\title{
Can we consider the Arctic Oscillation independently from the Barents Oscillation?
}

\author{
L.-Bruno Tremblay \\ Lamont Doherty Earth Observatory of Columbia University, Palisades, NY
}

\begin{abstract}
An EOF analysis of a constructed time series mimicking the Northern Hemisphere SLP variability of the last 50 years shows that the Barents Oscillation (BO) appears as a means to represent the sudden eastward shift of the northern center of action associated with the Arctic Oscillation (AO) observed in the mid-seventies. This sudden shift (non-stationarity) appears in an EOF analysis as a step change in the relative phase between the principal components associated with the EOFs of the AO and BO. The results also show that an EOF analysis of a constant amplitude signal can produce artificial trends and/or amplitude changes in the principal component associated with a given mode (eg. AO) when such non-stationarities are present in the signal. In this case, different modes of variability represented by EOF's cannot be considered independently from one another. In the example presented, although the principal components are completely uncorrelated from one another, perfect correlation and anti-correlation are present in the first and second parts of the time series respectively.
\end{abstract}

\section{Introduction}

Early on, the North Atlantic (NAO) and North Pacific (NPO) Oscillations were identified as two important modes of variability in the Northern Hemisphere (NH). These modes clearly stand out in an EOF analysis of SLP in the NA and NP sectors and manifest themselves as north-south dipoles with a sub-polar and a mid-latitude center [e.g., Wallace and Gutzler, 1981; Hurrell, 1995; Rogers 1990]. More recently, Thompson and Wallace [1998] introduced the Arctic Oscillation as the leading EOF in SLP over the entire $\mathrm{NH}$. Although the $\mathrm{AO}$ and the NAO share many common features, the $A O$ has a northern center of action that covers more of the Arctic and an additional weaker center in the North Pacific, giving it a more zonally symmetric appearance. From a non-linear principal component analysis, "Yonahan et al. $[2000]$ show that the Northern Hemisphere rriability is better characterized by an Arctic-Eurasian os(llation which is occasionally replaced by a split-flow configuration in the North Atlantic, with the AO representing some average between the two states. In the framework of the Arctic Oscillation, Skeie [2000] introduced the Barents Oscillation - the second EOF of the NH monthly mean SLP variability north of $25^{\circ} \mathrm{N}$ - which influences, among other things, the Eurasian surface air temperatures (SATs) in much the same way as the AO pattern, and the sensible heat fluxes (SHFs) in the Nordic seas. Skeie notes however, that an analysis of NCAR SLP data for 1899-1947

Copyright 2001 by the American Geophysical Union.

Paper number 2001GL013740.

0094-8276/01/2001GL013740\$05.00
[Skeie, 2000] does not reveal the presence of the BO pattern. In this note, an alternate interpretation of the $\mathrm{BO}$ mode of variability is presented, and more generally, the importance of considering separate statistical modes of variability together, for the analysis of certain climate signals, is shown.

\section{The problem}

In the context of a study of the link between the sea ice export through Fram Strait and the North Atlantic Oscillation (NAO), Hilmer and Jung [2000] described a secular change in the position of the northern center of action associated with the NAO pattern around 1976. This change is apparent from looking at the first mode of variability of the NH SLP for the periods 1949-76 and 1977-99 ${ }^{1}$ shown in Fig. 1a and $1 \mathrm{~b}$ (Hilmer and Jung obtained similar results from a linear regression of the NAO index onto the winter SLP anomaly field for the two sub-periods). This change was also reported by Cavalieri and Hakkinen [2001], who noticed a shift in the mean phase of planetary-scale SLP wave number 1 and 2 in the early seventies, and by Armstrong et al. [2001], who noticed a step change in the correlation between the NAO index and the winter sea ice concentration anomalies in the Laptev and Kara seas at around the same time. In the following we will refer to this change as a regime shift.

Interestingly, the spatial pattern of this regime shift can be reproduced from an EOF analysis of the entire time period (1949-99). This is shown in Fig. 2b where the third EOF $^{2}$ of the 1949-99 time period is seen to be similar to the difference map between the first mode of each of the two sub-periods (Fig. 1c). The first EOF for the entire time period (Fig. 2a) is the AO pattern, with a northern low pressure cell located in some average position between the two sub-period low pressure cells (see Fig. 1a and 1b). The third mode of atmospheric variability, appearing as an eastwest dipole centered over the east Greenland current, was named the Barents Oscillation (B0) by Skeie $[2000]^{3}$ since the main center of action is in the Barents/Norwegian seas. Although the spatial pattern of this regime shift is picked up by the third EOF, there are no apparent irregularities in the principal component associated with this mode in the midseventies (Fig. 2c, dashed line). The question we are asking is the following: Given that the nature of the atmospheric

\footnotetext{
${ }^{1}$ These modes'were obtained from an EOF analysis of winter mean (DJFM) SLP anomaly data (poleward of $25 \mathrm{~N}$ ) from the NCEP reanalysis.

${ }^{2}$ The spatial pattern of the second EOF is a dipole with a strong center in the northern North Pacific and a weaker center in the western Arctic (see also Yi et al. [1999]).

${ }^{3}$ In Skeie [2000], the Barents Oscillation appears as the second mode of variability as the time period analyzed is different (195899 as opposed to 1949-99).
} 
circulation in the North Atlantic clearly changed in the midseventies (Fig. 1a and 1b) and that this change (Fig. 1c) is well captured by the third EOF of the entire time period (Fig. 2b), why is there no apparent sign of this change in the principal component time series (Fig. 2c) associated with this mode in the mid-seventies? Or, how does an EOF analysis capture such secular changes present within a time series?

\section{Toy model of the North Atlantic atmospheric variability}

To answer this question, a simple toy model of the North Atlantic atmospheric variability is constructed. Consider a simplified representation of the NAO variability observed over the last 5 decades where the Azores High and Icelandic Low are represented by two squares ( 3 grid cells wide by 3 grid cells long), with the first one to the south of the other, and each square oscillating between a state of +1 and -1 from year to year (see Fig. 3). In analogy with the observed
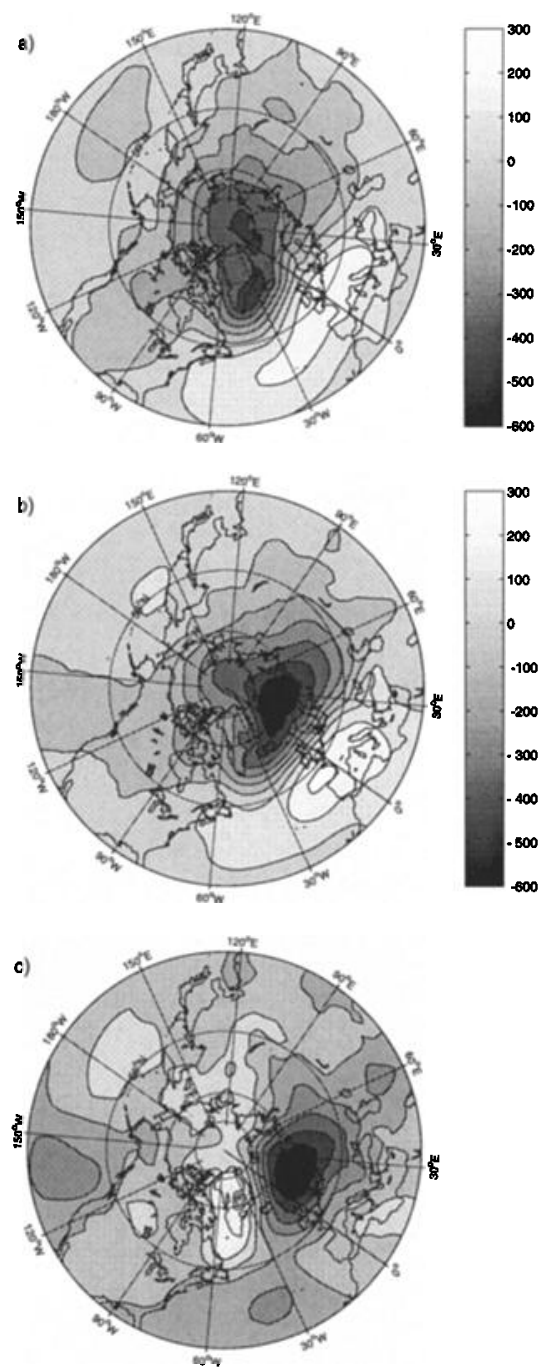

Figure 1. First EOF of the SLP anomaly field for the 1949-76 (a) and 1977-99 (b) time periods, and the difference map between the two (c). In (a) and (b) the EOF were scaled by the standard deviation of their respective principal component. The variance explained by the two modes is $30 \%$ and $34 \%$ respectively.
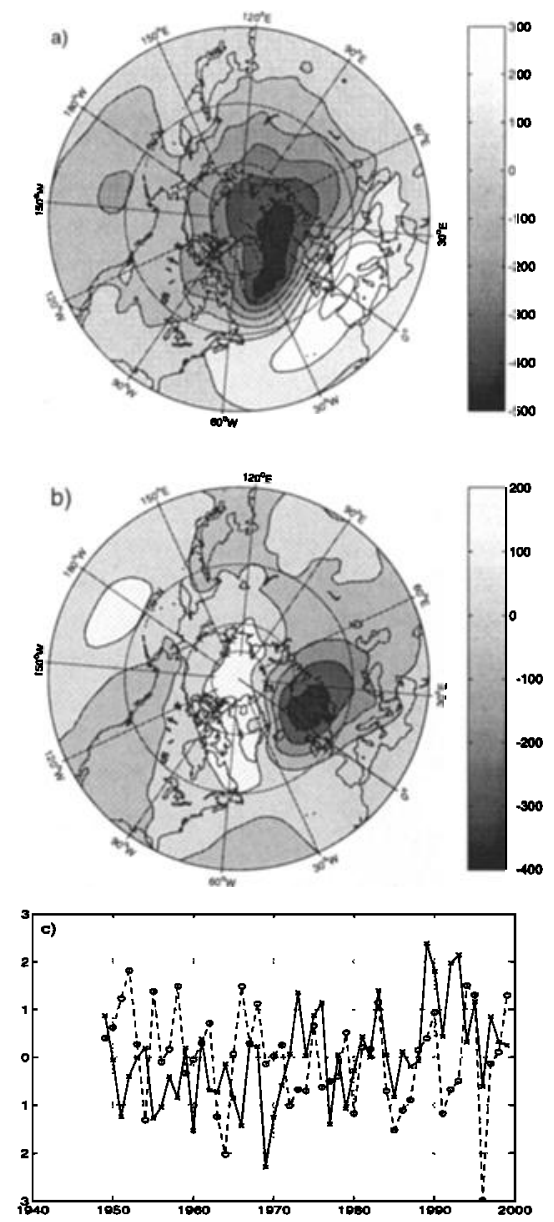

Figure 2. First (a) and third (b) EOFs and principal components (c) of the SLP anomaly field for the 1949-99. The first (solid line with $\mathrm{x}$-mark symbols) and third (dashed line with circle symbols) $\mathrm{PCs}$ represent the $\mathrm{AO}$ and $\mathrm{BO}$ respectively. The variance explained by the first three modes of variability is $32 \%, 16 \%$ and $9 \%$. These modes are well separated according to the criteria of North et al. [2000].

North Atlantic variability (Fig $1 \mathrm{a}$ and 1b), the Icelandic Low center of action in this simple model, is positioned to the north-west of the Azores High for the first 20 years with a sudden change to its north-east for the last 20 years. To this end, a $40 \times 81$ matrix (40 years of data on a $9 \times 9$ physical domain) of zeros, plus ones and minus ones was constructed and analyzed using EOF analysis (zeros were used everywhere but at the centers of action).

In this simple model the first two modes of variability represent the Arctic Oscillation and the Barents Oscillation (Fig. 4a and 4c) and explain $100 \%$ of the total variance ( $83.3 \%$ and $16.7 \%$ respectively). As seen from the principal components associated with these two modes of variability (Fig. 4e), the sudden change in the position of the northern center of action (idealized Icelandic Low) does not manifest itself as a step change in the magnitude of the principal components ( $\mathrm{AO}$ or $\mathrm{BO}$ indices) but rather as a step change in the relative phase between the two modes of variability. In the first 20 years, the $A O$ and $B O$ time series are 180 degrees out of phase and a constructive interference in the north-west and destructive interference in the north-east of the domain is present. At year 21, there is a step change 


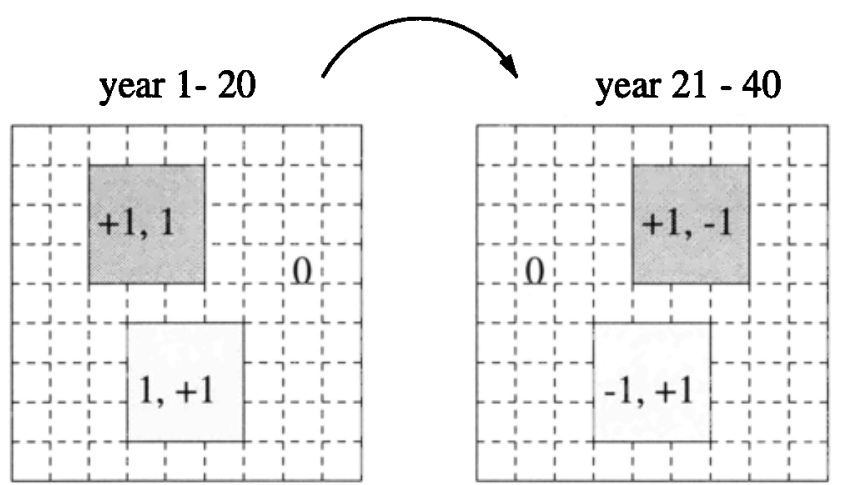

Figure 3. Idealized SLP pattern analyzed using EOF analysis. In this toy model, a seesaw in SLP $(+1$ and -1$)$ is imposed for 40 years with a sudden eastward shift in the northern cell position after year 20.

in the relative phase of the two time series and the reverse situation is observed. This shows how an EOF analysis decomposes a non-stationary process into basic modes that are orthogonal to each other. As expected, the correlation coefficient between the two principal components for the entire time period is zero; however it is equal to -1 and +1 for the first and last 20 years of the time series respectively. The Barents Oscillation therefore only appears in this analysis as a way to represent the non-stationarity of the AO spatial pattern.

In addition, changes in the amplitude of the principal components at the time when the sudden eastward shift is imposed, can be produced in this idealized NAO oscillation without changing the amplitude of the original SLP signal. This is done simply by introducing spatial or temporal asymmetries in the problem. For instance, increasing the length of time of the second regime (relative to the first one), or increasing the area of the low pressure cell in the northeast of the domain for the last 20 years ${ }^{4}$, without changing the amplitude of the seesaw (i.e. +1 and -1 ), causes an increase (decrease) in amplitude in the first (second) principal component ( $\mathrm{AO}$ or $\mathrm{BO}$ index) at the step along with a phase change. The results from the first scenario are shown in Fig. $4 \mathrm{~b}, 4 \mathrm{~d}$ and $4 \mathrm{f}$ for a similar 40-year data set but, where the shift between the two modes of oscillation is imposed after year 10 instead of year 20 (the second scenario gives analogous results). Note that the spatial patterns in this case are very similar to the previous case except for the fact that the northern center has shifted east in the first EOF and a weak (not significant) southern center has now appeared in the second EOF.

This analysis shows that a regime shift, such as the one observed in the mid-seventies, can only be seen (when using linear EOF analysis) by looking for a relative phase shift between two different principal components, and that changes in amplitude in the $\mathrm{AO}$ and $\mathrm{BO}$ indices can be the result of spatial or temporal asymmetries between different regimes rather than actual changes in the amplitude of the seesaw in the original SLP time series. The change in amplitude at the step will be a function of the partition of energy between the two modes. In this case the amplitude change is small

\footnotetext{
${ }^{4}$ This is apparent from Fig $1 b$ which shows a northern cell that covers a much larger area including the Barents, Norwegian, Iceland and Greenland seas.
}
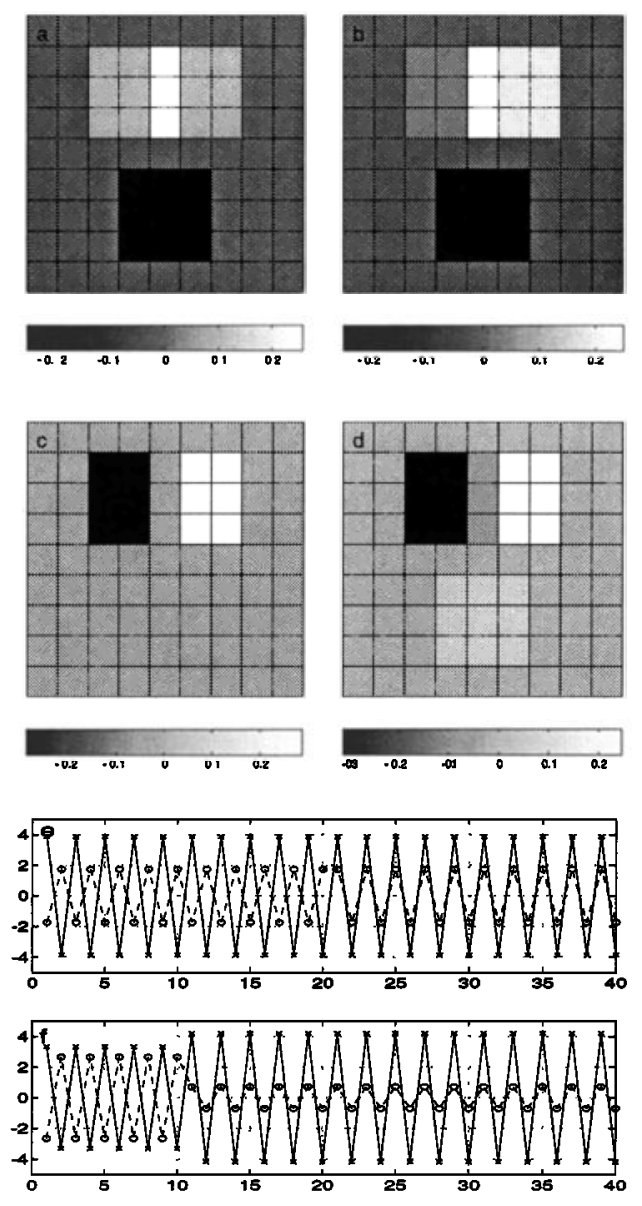

Figure 4. EOFs and PCs for two idealized NAO variability scenarios. In the first scenario ( $a, c$ and $e$ ), a seesaw in SLP is imposed with a sudden eastward shift in the northern cell position after year 20; in the second (b, $d$ and $f$ ), the shift occurs after year 10. The first PC in both (e) and (f) is shown as a solid line with $\mathrm{x}$-mark symbols and the second $\mathrm{PC}$, as a dashed line with circle symbols. In the first scenario, the first and second modes of variability explain $83.3 \%$ and $16.7 \%$ of the total variance respectively; in the second scenario, they explain $88.2 \%$ and $11.8 \%$.

as the first and second EOFs account for $83.3 \%$ and $16.7 \%$ of the total variance. In the case of the $\mathrm{AO}$ and $\mathrm{BO}$ pattern, this effect might be more important as the fraction of the total variance is of the same order of magnitude (32\% and $9 \%$ respectively). In general, the results suggest that in the presence of non-stationarities, different modes of variability (although orthogonal to each other) should be considered together. Alternatively, EOF analysis can be used with a moving time-window on the original data to identify changes in the basic modes of variability.

When looking at the principal components of the actual SLP variability (see Fig. 2c, where PC1 and PC3 are plotted together), the phase shift is apparent from the $A O$ and BO time series which have a correlation of -0.29 for the 1949-76 time period and 0.40 for the 1977-99 time period. The anti-correlation for 1949-76 is lower than for 1977-99 as some BO pattern is present in that sub-period ${ }^{5}$. From

\footnotetext{
${ }^{5}$ The BO appears as the third EOF in the 1949-76 time period. However, it is not well separated from the second EOF according to the criterion of North et al. [1982]. For 1977-99, the BO pattern does not appear in the first four EOFs.
} 
Fig. 2c, an increase in amplitude of the AO index around 1970, accompanied by a decrease in amplitude of the BO index, is also clearly visible. A comparison between the $\mathrm{AO}$ and NAO time series (not shown here) shows a larger amplitude increase in the AO index in 1970 than does the NAO index (which is based on a SLP difference rather than a principal component associated with a given mode of variability). This analysis suggests that this exaggerated amplitude change in the AO index in 1970 could be an artifact of the EOF analysis method.

Acknowledgments. B.T. is greatful to A. Kaplan, P. Skeie and G. Flato for useful discussions and to A. Armstrong and S.A. Venegas for providing assistance with the statistical analysis. As well, I would like to thank A. Armstrong, S.A. Venegas, L.A. Mysak and an anonymous reviewer for useful comments on the manuscript. This work was supported by the International Arctic Research Center (IARC) under grant \#PF904713 and the National Science Foundation, under grant OPP98-18711.

\section{References}

Armstrong, A.E., L.-B. Tremblay and L.A. Mysak, A data-model intercomparison study of Arctic sea-ice variability, Clzmate Dynamics, submitted, 2001.

Cavalieri, D.J. and S. Hakkinen, Arctic climate and atmospheric planetary waves, Geophys. Res. Lett., 28, 791-794, 2001.

Hilmer, M. and T. Jung, Evidence for a recent change in the link between the North Atlantic Oscillation and Arctic sea ice export, Geophys. Res. Lett., 27, 989-992, 2000.
Hurrell, J.W., Decadal trends in the North Atlantic Oscillation: regional temperatures and precipitation, Science, 269, 676$679,1995$.

Monahan, A.H., J.C. Fyfe and G.M. Flato, A regime view of Northern Hemisphere atmopsheric variability and change under global warming, Geophys. Res. Lett., 27, 1139-1142, 2000.

Rogers, J.C., Patterns of low-frequency monthly sea level pressure variability (1899-1986) and associated wave cyclone frequencies, Journal of Climate, 3, 1364-1379, 1990.

Skeie, P., Meridional flow variability over Nordic seas in the Arctic Oscillation framework, Geophys. Res. Lett., 27, 2569-2572, 2000.

Thompson, D.W.J. and J.M. Wallace, The Arctic Oscillation signature in the wintertime geopotential height and temperature fields, Geophys. Res. Lett., 25, 1297-1300, 1998.

Wallace, J.M. and D.S. Gutzler, Teleconnections in the geopotential height field during the Northern Hemisphere winter, Monthly Weather Review, 109, 784-812, 1981.

Yi, D., L.A. Mysak and S.A. Venegas, Decadal-to-interdecadal fluctuations of Arctic sea-ice cover and the atmospheric circulation during 1954-1994, Atmosphere-Ocean, 37, 389-415, 1999.

B. Tremblay, Lamont Doherty Earth Observatory of Columbia University, Rt 9W, Palisades, NY 10964-8000, USA. (email: trem blay@ldeo.columbia.edu)

(Received July 6, 2001; revised August 29, 2001; accepted September 10, 2001.) 